\title{
The risk analysis of pseudolite and satellite navigation system
}

\author{
J. Taufer \& L. Bazant \\ TUCD - Laboratory of Intelligent Systems, Czech Railways, \\ Czech Republic
}

\begin{abstract}
The modern navigation and positioning methods are increasingly used in safety related applications. These methods are able to pursue navigation functions with very high accuracy and also with high frequency. Safety applications also require risk analysis of the methods used. The indivisible part of such an analysis must be integrity monitoring (IM) of the positioning solution.

Today, the meaning of satellite navigation system (GNSS) increases. But for many applications, especially for transport systems, the satellite navigation in itself may not be sufficient due to the visibility of only a small number of satellites, or none at all, at the time of a safety critical event, high safety requirements etc. In this case, the system must be completed with another system. Most often it's the system of inertial sensors, but it is also possible to use the system of ground transmitters of the satellite signal - the system of pseudolites (PL's). Such a system is "ground equivalent" to the satellites system, but it has certain specifics, namely accurately defined and fixed position of signal transmitters, warranted availability in the required area. These specifics are significant for design of IM.

The applications based on satellites and PL's navigation system can be designed in two ways. The first way is a direct combination of signals from satellites and PL's in one receiver and one solution of position together with one risk analysis.

It assumes the time synchronization of GNSS and PL's system (synchr. PL's according to GNSS time). The advantage of this way is the possibility of using so-called "Assisted GPS" (A-GPS). The second way is using two independent channels - one for PL's system and the second for GNSS and a combination of both independent solutions and also their risk analysis.
\end{abstract}

Keywords: pseudolites, safe positioning, integrity monitoring. 


\section{Introduction}

Many safety related applications (e.g. aircraft precision approach or landing, rail movement etc.) require very high integrity. The most important information for such applications are accurate and mainly safe determination of their position. In such an operational environment it is important to exploit all available information which may be a common problem.

Safety applications based on satellite navigation fail when the receiver used has not a sufficient number of available satellites. Therefore, it's necessary to use several independent resources of information, particularly in situations of critical time or in a critical area. For example, the system of inertial sensors performs only relative measurements and such a system in itself alone is not usable over a long time, because errors of these sensors will be accumulated in time. It seems that systems providing absolute measurements of position will be preferable. The possibility of an easy combination with GNSS can also be an advantage. Such system can be, for example, a system of pseudolites (PL's).

The most important values for IM, which provides the necessary information to issue timely warnings if the position solution is not used, is the accuracy of this solution, the Integrity Risk (IR) and the Continuity of Service (CoS). The IR is a probability of hazardous misleading information $\left(\mathrm{P}_{\mathrm{HMI}}\right)$. This is a probability for which the user should have been warned. The $\operatorname{CoS}$ is a probability that the user will have the integrity information continuously through the required time interval. Continuity Risk (CR) is a complement of $\mathrm{CoS}$ to unit (CR $=1-\mathrm{CoS})$. It can be caused by IM alerts, by loss or failure of IM messages or by failure of the receiver itself. In terms of safety, it's possible to perform a safety function based on a position solution, if the IR and CR conditions are simultaneously satisfied. If the IR exceeds the specified value or the IM is not available, the application has to immediately stop all safety functions and operations. If the IR is below the specified value, but the CR exceeds the allowed value, the application is not allowed to start to use the position for safety operation but it's allowed to continue to use it for the running of the operation.

The IR can be calculated in two ways. Firstly, the $\mathrm{P}_{\mathrm{HMI}}$ can be directly calculated at the maximum allowed position error - Alert Limit and it will be compared with maximum allowed IR or secondly, the calculation of Protection Level at the given maximum value of the IR, which has to be below the required Alert Limit.

\section{PL's dislocation}

For the effectivity of PL's positioning a good PL's signal availability must be ensured at the rover receiver with respect to proper level of received signal, minimization of signal multipath occurrence probability etc. The reference station must be located in the point with minimum impact of signal failures on the position determination and must provide corrections and the integrity messages subsequently transmitted over the whole area. This point must ensure good availability of GNSS, if it is also used. 
The dislocation of PL's transmitters with the reference and mobile receivers is shown in fig. 1. The reference station is composed from the receiver, computer unit and the radio station that receives signals from PL's and simultaneously synchronizes PL's system, and also computes the IR for assumed usage area, and provides an integrity message to a mobile receiver. In addition, it's possible to generate differential or phase-carrier corrections and transmit them to the mobile receiver. In this case, it needs to pursue the monitor of correction integrity. In virtue of a limited usable range of power level of signals received from the PL's and thus relatively small area of usage, it can be assumed that the same conditions of the signal propagation exist in the whole area with a single exception - the possibility of multipath occurrence. But, it can be minimized by means of suitable transmitter dislocation, using a suitable antenna for the mobile receiver and PL's etc.

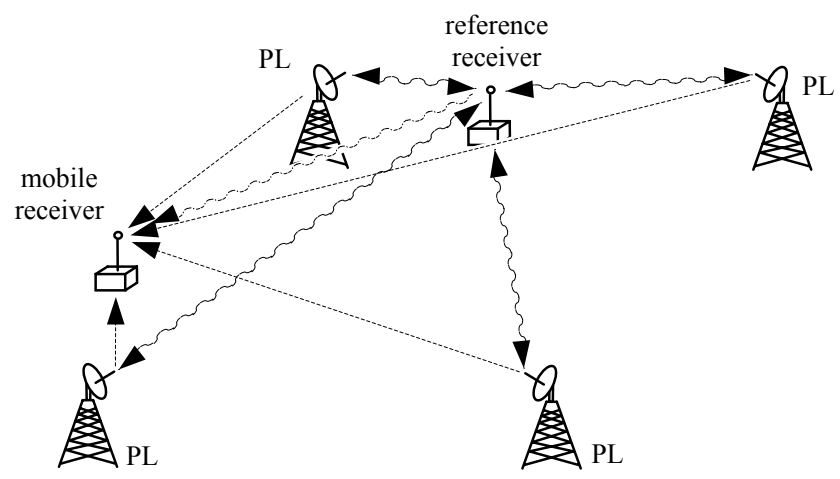

Figure 1: The dislocation of PL's, reference and mobile station.

\section{Integrity monitoring at the reference and mobile receiver}

The first task of the reference station is the PL's system time synchronization. According to the PL's system conception it can be synchronized along one of PL's or by the external (GNSS) time.

It's possible to determine the position of the reference receiver with almost absolute accuracy, because the reference station has a fixed position. The same applies to positions of PL's transmitters (in contrast to system of GNSS satellites). This fact can be used with advantage in the design of IR at the reference station.

The same method for the computation of the position, which is used on the mobile receiver (usually last square method), must be also used for the computation of the reference station position. The user must be able to determine (or leastways estimate) the IR for the computed position of the mobile receiver also from the reference station (on the assumption that conditions of signal reception are the same in whole area). If conditions of signal reception in position of the mobile receiver are different from reference station (multipath,...) 
or the method of processing is different, then the relevant probability must be included into the IR.

The vector of measured pseudoranges between the receiver and transmitters is

where

$$
\boldsymbol{r}=\boldsymbol{R}+\mathrm{c} . \delta \mathrm{t}+\boldsymbol{v}
$$

$$
\begin{array}{ll}
\boldsymbol{R} & \text { is vector of true pseudoranges } \\
\mathrm{c} . \delta \mathrm{t} & \text { is clock offset multiplied by speed of light } \\
\boldsymbol{v} & \text { is vector of measurement errors }
\end{array}
$$

The Taylor expansion linearization of this equation can be describe as

where

$$
\boldsymbol{r}=\boldsymbol{R}_{\boldsymbol{0}}+\boldsymbol{H} . \delta \boldsymbol{x}+\boldsymbol{L R}+\mathrm{c} . \delta \mathrm{t}+\boldsymbol{v}
$$

$$
\begin{aligned}
& \boldsymbol{H}=\quad-\left(x_{t 11}-x_{r 01}\right) / R_{01} \quad-\left(x_{t 12}-x_{r 02}\right) / R_{01} \quad-\left(x_{t 13}-x_{r 03}\right) / R_{01} \\
& -\left(\begin{array}{lll}
\left.x_{t 21}-x_{r 01}\right) / R_{02} & -\left(x_{t 22}-x_{r 02}\right) / R_{02} & -\left(x_{t 23}-x_{r 03}\right) / R_{02}
\end{array}\right. \\
& \begin{array}{lcc}
\cdots & \cdots & \cdots \\
-\left(x_{t N 1}-x_{r 01}\right) / R_{0 N} & -\left(x_{t N 2}-x_{r 02}\right) / R_{0 N} & -\left(x_{t N 3^{-}} x_{r 03}\right) / R_{0 N}
\end{array} \\
& \delta \boldsymbol{x}=\left(\boldsymbol{H}^{T} \cdot \boldsymbol{P}^{-1} \cdot \boldsymbol{H}\right)^{-1} \cdot \boldsymbol{H}^{T} \cdot \boldsymbol{P}^{-1} \cdot\left(\boldsymbol{r}-\boldsymbol{R}_{\boldsymbol{0}}\right) \\
& \boldsymbol{P} \text { is covariance matrix of observations }(\mathrm{NxN}) \\
& \boldsymbol{R}_{0} \quad \text { is vector of est. pseudoranges between receiver and transmitters }(1 \mathrm{xN}) \\
& \boldsymbol{x}_{t i} \quad \text { is vector of 3D-cartesian coordinates of } i \text {-transmitter position (1x3) } \\
& \boldsymbol{x}_{r 0} \quad \text { is vector of } 3 \mathrm{D} \text {-cartesian coordinates of est. position of receiver }(1 \times 3) \\
& \boldsymbol{L} \boldsymbol{R} \text { is vector of Lagrange residuals }(1 \mathrm{xN}) \\
& i \quad \text { is number of transmitter }(1 \ldots \mathrm{N})
\end{aligned}
$$

The computed position $\boldsymbol{x}_{\boldsymbol{C}}$ is the sum of first estimation $\boldsymbol{x}_{\boldsymbol{0}}$ and residual $\delta \boldsymbol{x}$ and the difference between computed and known position is the position error

$$
\begin{aligned}
& \varepsilon=x_{C}-x= \\
& =x_{0}+\delta x-x
\end{aligned}
$$

This position error in PL's positioning system can be caused by

$$
\begin{array}{ll}
\text { - } & \text { noise, stochastic error of received signal (pseudorange) } \\
\text { - } & \text { signal propagation conditions in atmosphere } \\
\text { - } & \text { faulty clock offset determination, clock bias } \\
\text { - } & \text { multipath propagation }
\end{array}
$$

Due to the relatively small usage area, the noise, atmospheric conditions and clock determination are common to all receivers. The method in itself should not cause any errors, or if there are any, then only those that can be accurately determined from the reference station (the used method in itself should only 
eliminate the occurred errors). Some predictive iterative filters (often used Kalman filters) can cause the error, although processed data are without errors.

The task of the reference station must be to determine the IR coverage of the whole given area, because the position of the mobile receiver (or receivers) cannot be known.

In every point of usage area that can cause a dangerous error (the position error $\varepsilon$ greater than allowed value) suffices the different value of pseudoranges error $v$. One needs to find the point in the usage area with the least value of pseudoranges error, so-called least bias $\boldsymbol{b}_{\text {min }}$, which causes the dangerous position error (the point, where the probability of the dangerous error occurrence owing to pseudorange error $v$ is largest). Therefore, there must exist the exact relation between the pseudorange error and error of the computed position. If the error of pseudoranges is under the least bias, the required conditions of the maximum IR will be executed in the whole usage area and only risk of multipath propagation exists for the mobile receiver. Usually, for the user, only some fraction of the position error is important (or only the projection to horizontal or vertical plane). As the user usually doesn't know which plane will be important to him, he needs to assume the worst-case

$$
\varepsilon_{p o s}=\left(\varepsilon_{1}^{2}+\varepsilon_{2}^{2}+\varepsilon_{3}^{2}\right)^{1 / 2}
$$

The position error can be written as $\varepsilon=f(x, v)$, according to inverse $v=\phi(x, \varepsilon)$ the least bias is

where

$$
\boldsymbol{b}_{\text {min }}=\min \{\boldsymbol{v}=\phi(\boldsymbol{x}, \boldsymbol{A P E})\}_{\boldsymbol{x} \in U A}
$$

$\boldsymbol{A P E}$ is allowed position error

$U A$ is usage area.

The propagation of the observations covariance matrix $\boldsymbol{P}$ by means of the matrix $\boldsymbol{H}$ is the covariance matrix of position error

$$
\begin{aligned}
\boldsymbol{P}_{\varepsilon}=\left(\left(\boldsymbol{H}^{T} \cdot \boldsymbol{P}^{-1} \cdot \boldsymbol{H}\right)^{-1} \cdot \boldsymbol{H}^{T} \cdot \boldsymbol{P}^{-1}\right) \cdot \boldsymbol{P}^{-1} \cdot\left(\left(\boldsymbol{H}^{T} \cdot \boldsymbol{P}^{-1} \cdot \boldsymbol{H}\right)^{-1} \cdot \boldsymbol{H}^{T} \cdot \boldsymbol{P}^{-1}\right)^{T}= \\
=\left(\boldsymbol{H}^{T} \cdot \boldsymbol{P}^{-1} \cdot \boldsymbol{H}\right)^{-1}
\end{aligned}
$$

It's possible to perform the simplification of this matrix in the elliptical shape to the diagonal circular shape with worst value

$$
\sigma^{2}=\max \left\{\left(P_{\varepsilon i, i}+P_{\varepsilon i, j}\right) / 2+\left[\left(P_{\varepsilon i, i}+P_{\varepsilon j, j}\right)^{2} / 4+P_{\varepsilon i, j}\right]^{1 / 2}\right\}_{i \neq j}
$$

For this system, two cases come on force. The failure-free case $\left(f f_{c}\right)$, where the position error $\boldsymbol{\varepsilon}_{\boldsymbol{p} \boldsymbol{s}}$ has normal distribution with zero mean value $\boldsymbol{N}\left(\boldsymbol{0}, \boldsymbol{P}_{\varepsilon}\right)$, and faulty case $(f c)$, where there is an assumption of the non-zero mean of position error distribution $\boldsymbol{\mu}$. The $\boldsymbol{P}_{H M I}$ in the $f f c$ is the product of probability that signals from all transmitters with a zero mean value that are in allowed limits, which cannot cause a dangerous position error and exceeding of the required IR probability in this case. The $\boldsymbol{P}_{H M I}$ in $f_{C}$ is given by the product of probability when signals have non-zero mean value and the relevant IR. Whole IR is a sum of both cases. 
On the fig. 2 is shown the determination of probabilities of the dangerous failures occurence in the signal of i-transmitter, which is related to worst-point of usage area - $\boldsymbol{P}_{\text {signal,inAPE, } i}$ and $\boldsymbol{P}_{\text {signal,outAPE, } i}$. The failure of signal, which could cause the error of pseudorange $\boldsymbol{v}$ and subsequently the dangerous position error $\varepsilon_{\text {pos }}$ can be positive or negative. With regard to dislocation of pseudolites, values of $\boldsymbol{A P E}, \boldsymbol{P}$ and size of the usage area, the probability that pseudorange error from some transmitter will be smaller than negative $b_{\min }$ is insignificant. Therefore, the probabilities $\boldsymbol{P}_{\text {signal,inAPE, } i}$ and $\boldsymbol{P}_{\text {signal,out } A P E, i}$ can be written as

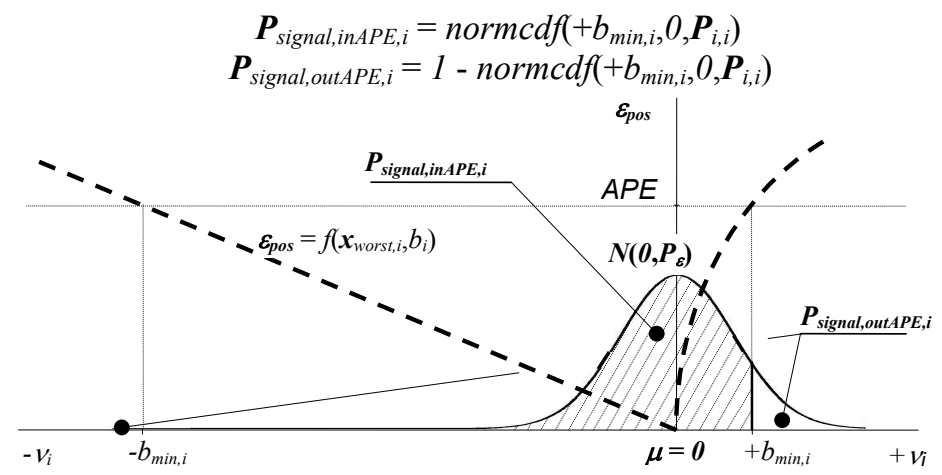

Figure 2: $\quad$ Probabilities $\boldsymbol{P}_{\text {signal,inAPE, } i}$ and $\boldsymbol{P}_{\text {signal,outAPE,i}}$ for the $f f c$ case.

Relevant probabilities of IR for $f f c$ and $f_{c}$ cases can be written by the following term, whereas for simplification of the $f c$ case it's possible to allow for the worst-case with positive least bias $+b_{\min }$.

$$
\begin{aligned}
& \boldsymbol{P}_{I R, f f c, i}=\left(1-\chi^{2} c d f\left(\boldsymbol{A P E} \boldsymbol{E}^{2} / \sigma_{i, f f c}^{2}, 3\right)\right) / 2 \\
& \boldsymbol{P}_{I R, f c, I}=\left(1-\chi_{\text {non-central }}^{2} c d f\left(\boldsymbol{A} \boldsymbol{P} \boldsymbol{E}^{2} / \sigma_{i, f c}^{2}, 3, \delta_{i}\right)\right)
\end{aligned}
$$

where non-centrality parameter is $\delta_{i}=\boldsymbol{\beta}_{\min , i}{ }^{T} . \boldsymbol{P}^{-1}{ }_{\varepsilon f f .} \boldsymbol{\beta}_{\text {min }, i}$ and $\boldsymbol{\beta}_{\text {min }, i}=\left(\boldsymbol{H}^{T} . \boldsymbol{P}_{f c}^{-l} \cdot \boldsymbol{H}\right)^{-1} . \boldsymbol{H}^{T} . \boldsymbol{P}_{f c}^{-1} \cdot \boldsymbol{\mu}_{l}$

For the total IR in usage area can be written the relation

Where

$$
\begin{gathered}
\boldsymbol{P}_{H M I, i}=\boldsymbol{P}_{\text {signal, inAPE, ffc, } i} \boldsymbol{P}_{I R, f f c, i}+\boldsymbol{P}_{\text {signal, inAPE }, f c, i} \cdot \boldsymbol{P}_{I R, f c, i} \\
\boldsymbol{P}_{H M I}=1-\prod_{\mathrm{i}=1 . . \mathrm{n}}\left(1-\boldsymbol{P}_{H M I, i}\right)
\end{gathered}
$$

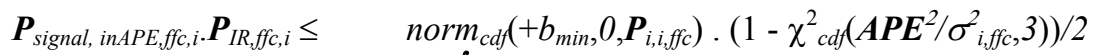

$$
\begin{aligned}
& \boldsymbol{P}_{\text {signal, inAPE }, f c, i} . \boldsymbol{P}_{I R, f c, i} \leq \int_{\mu i \in \boldsymbol{R}-\{0\}} \boldsymbol{P}_{\text {signal,inAPE }, f c, \mu i} . \boldsymbol{P}_{I R, f c, \mu i} . \mathrm{d} \mu_{i}
\end{aligned}
$$

and there

$$
\begin{aligned}
& \boldsymbol{P}_{\text {signal,inAPE }, f c, \mu i}=\operatorname{norm}_{p d f}\left(\mu_{i}, 0, \boldsymbol{P}_{i, i, f f c}\right) \cdot\left(\operatorname{norm}_{c d f}\left(+b_{m i n}, \mu_{i}, \boldsymbol{P}_{i, i, f c}\right)-\operatorname{norm}_{c d f}\left(-b_{m i n}, \mu_{i}, \boldsymbol{P}_{i, i, f c}\right)\right.
\end{aligned}
$$

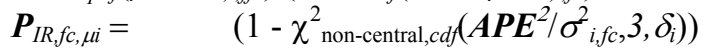


In the probability of a false alarm, there is a probability that the system will issue the warning, although the user should not have been warned because the system is without dangerous failures. For the determination of the false alarm probability $\left(\mathrm{P}_{\mathrm{FA}}\right)$ it's possible to use the process analogic to $\mathrm{P}_{H M I}$ determination.

Where

$$
\begin{gathered}
\boldsymbol{P}_{F A, i}=\boldsymbol{P}_{F A, f f, i}+\boldsymbol{P}_{F A, f c, i} \\
\boldsymbol{P}_{F A}=1-\prod_{\mathrm{i}=1 . \mathrm{n}}\left(1-\boldsymbol{P}_{F A, i}\right)
\end{gathered}
$$

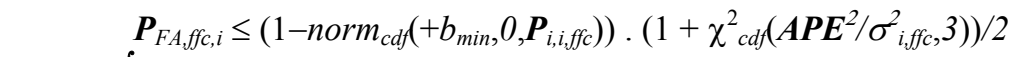

$$
\begin{aligned}
& \boldsymbol{P}_{F A, f c, i} \leq \int_{\mu i \in \boldsymbol{R}-\{0\}} \boldsymbol{P}_{s, \text { outAPE,fc, } \mu i} . \boldsymbol{P}_{A, f c, \mu i} . \mathrm{d} \mu_{i} \\
& \text { and there } \\
& \boldsymbol{P}_{s, \text { outAPE } f f c, \mu i}=\operatorname{norm}_{p d f}\left(\mu_{i}, 0, \boldsymbol{P}_{i, i, f f c}\right) .\left(1-\text { norm }_{c d f}\left(-b_{\text {min }}, \mu_{i}, \boldsymbol{P}_{i, i, f c}\right)-\text { norm }_{c d f}\left(+b_{m i n}, \mu_{i c}, \boldsymbol{P}_{i, i, f c}\right)\right)
\end{aligned}
$$

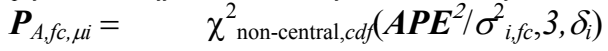

The calculation of a false and true alarm probability is important for the determination of the CoS.

$$
\begin{gathered}
\boldsymbol{P}_{A, i} \leq \int_{\mu i \in \boldsymbol{R}-\left\{0_{0} ;\right.} \operatorname{norm}_{p d d}\left(\mu_{i}, 0, \boldsymbol{P}_{i, i, f c c}\right) . \\
.\left(1-\operatorname{norm}_{c d f}\left(-b_{m i n}, \mu_{i c}, \boldsymbol{P}_{i, i, j c}\right)-\operatorname{norm}_{c d f}\left(+b_{m i n}, \mu_{i c}, \boldsymbol{P}_{i, i, f c}\right)\right) . \mathrm{d} \mu_{i} \\
\boldsymbol{P}_{A}=1-\prod_{\mathrm{i}=1 . \mathrm{n}}\left(1-\boldsymbol{P}_{A, i}\right)
\end{gathered}
$$

The mobile receiver doesn't know its position. Therefore, the IM must be pursued for the estimated position $\boldsymbol{x}_{r 0}$. The IR in the mobile receiver position must be lower then maximum IR of usage area computed from the reference station. Therefore it's possible to compare the computed IR and the IR obtained from the reference station. (Naturally, the possibility of the signal affected by multipath needs to be included.)

For achieving of a higher required safety condition, the system of pseudolites can be extended by the GNSS satellite system. There are two possible ways. The first one, the usage of two independent channels for position determination and subsequent comparison of both solutions. In this case, only the receiver antenna is common to both channels. The independent solution of position determination by means of the GNSS has a certain disadvantage. For the 3D-determination of the position, the receiver must receive at least four satellites. As the determination by means of PL's system is on the same principle, the combination of GNSS and PL's systems challenges to the common solution. If the PL's system is synchronized along the GNSS time, a small number of satellites (sometimes only one) could be included into the solution. This solution assumes that the mobile receiver knows the relevant satellite ephemeris data. Many safety applications and operations require prompt usability of positioning system and don't allow any waiting for sufficient number of satellites (in case of independent GNSS solution) or the reception of relevant ephemeris data from satellites (in case of the "cold start" of receiver). It seems that the optimal variant of system configuration will include the GNSS signal to one common solution together with signal from the PL's. 
The technical design of this common case enables also the usage of the "Assisted GPS" (A-GPS), which greatly reduces the "Time To First Fix" (TTFF), without other technical equipment. Due to usage of this technique, the time to first fix in case of a "hot start" may be shortened from 30-40 second up to 4 second. The reference station in such a system configuration receives signals from all satellites eventually visible from the mobile receiver at a given area and provides ephemeris data to the mobile receivers. The design of the IM for such combined system will be slightly complicated, because the position of satellites (satellite transmitters) is not accurately known.

\section{Technical equipment}

One of several different possibilities of the technical solution, which had been taken into account during the project proposal preparation, was usage of the "user-closed" system with PL's synchronized in some way to GPS time, transmitted modified codes in other frequency range and with higher dynamic receivers. The main advantages of such PL's systems consist in suppression of interference, near-far effect and possibility of combined navigation solution in GPS/PL's receiver. Unfortunately, the price of such a completed system (Novariant Terralite GPS/XPS) is out of range of our project budget.

A less expensive solution is PL's indoor system (unsynchronized to GPS time) based on signal generators which provide only GPS-like signals. Two manufacturers were found - Navicom and Space System Finland (SSF). While Navicom uses self-developed both PL's (NGS 2T) and receivers (NGS 2R), the SSF solution is based on self developed PL's and iTrax receivers from Fastrax. The decision to use SSF system was made.

The system consists of 4 PL's GPS-like signal generators that broadcast signal consisted of L1 carrier modulated by proper C/A code. The system includes the Master Control Station (MCS) which monitors the PL's functionality and ensures that the system is synchronized to PL's system time and stable. Due to this external solution and possibility to obtain GPS and PL's navigation and raw data the system as a whole remains open, which is important for our project task solution, namely the IM.

\section{Acknowledgements}

The work was supported by the Czech Science Foundation under contract No. 102/06/0052 and Transport Ministry of the Czech Republic under contract No. CG 743/037/520.

\section{References}

[1] Ober P., Integrity Monitoring, Delft University of Technology, 1996

[2] Pullen S., Pervan B., Enge P., Parkinson B., A Comprehensive Integrity Verification Architecture of On-Airport LAAS Category III Precision Landing, ION GPS 96, 1996 
[3] Hewitson S., Wang J., GNSS receiver autonomous integrity monitoring (RAIM) performance analysis, GPS Solutions, Volume 10, Number 3, 2006

[4] Djuknic G.M., Richton R.E., Geolocation and Assisted-GPS, Computer, Volume 34, 2001

[5] Braff R., Center for Advanced Aviation System Development, LAAS Performance for Terminal Area Navigation, www.mitre.org/work/ tech_papers/tech_papers/braff_lass/braff_lass.pdf 